\title{
Culicídeos associados a entrenós de bambu e bromélias, com ênfase em Aedes (Stegomyia) albopictus (Diptera, Culicidae) na Mata Atlântica, Paraná, Brasil
}

\author{
Allan Martins da Silva ${ }^{1}$; Valdecir Nunes ${ }^{2} \&$ José Lopes $^{3}$ \\ 1. Secretaria de Estado da Saúde do Paraná, 19ª Regional de Saúde, Rua Dr. Heráclio 90, 86400-000 Jacarezinho, PR, Brasil. \\ (allanms@ibest.com.br) \\ 2. Secretaria de Estado da Saúde do Paraná, $17^{a}$ Regional de Saúde, Rua Travessa Goiânia 152, 86.020-120 Londrina, PR, Brasil. \\ 3. Departamento de Biologia Animal e Vegetal, Universidade Estadual de Londrina, Campus Universitário, 86051-970 Londrina, \\ PR, Brasil. (jea@uel.br)
}

\begin{abstract}
Culicids associated with bamboo internodes and bromeliads, with emphasis on Aedes (Stegomyia) albopictus (Diptera, Culicidae) in the Atlantic Forest, Paraná, Brazil. Aedes (Stegomyia) albopictus (Skuse, 1894) larvae were recorded for the first time in the internodes of bamboo cuts found in the Atlantic Forest in the State of Paraná, Brazil. The bamboo, Bambusa sp., was introduced in the area of Imbucuí-Mirim town, across the municipal district of Paranaguá and towards the interior of the Atlantic Forest. A total of 251 larvae of Aedes albopictus (37.9\%), Culex (Microculex) spp. (13.9\%), Limatus durhami Theobald, 1901 (39.8\%), Toxorhynchites sp. (0.4\%) and Wyeomyia spp. (8.0\%) were collected from bamboo internodes filled with rain and from the terrestrial Bromeliaceae and epiphytas found near the bamboo bushes. Aedes albopictus was found in bamboo internodes containing 30 to $254 \mathrm{ml}$ of rain, sometimes coexisting with Li. durhami. Borders of forest ecosystems should be characterized as risk areas for arbovirosis transmission, since they sustain the circulation of vertebrates and arthropodal hematophagus, not only in the wild but also in the anthropic areas.
\end{abstract}

KEYWORDS. Culicidae, larvae, breeding site, Atlantic Forest, vectors ecology.

\section{INTRODUÇÃO}

Aedes (Stegomyia) albopictus (Skuse, 1894) é uma espécie exótica no Continente Americano (ForatTinI, 1986). De origem asiática, é vetor natural do vírus da dengue em áreas rurais e urbanas e do vírus da encefalite japonesa; adaptou-se ao ambiente antrópico e atualmente está dispersa por todas as regiões tropicais e temperadas no hemisfério ocidental. É capaz de veicular horizontalmente os quatro sorotipos do dengue e verticalmente, quando em circunstâncias experimentais, os sorotipos 1 e 4. Ainda em condições experimentais provou ser um bom transmissor de uma cepa do vírus da febre amarela, vírus da encefalite eqüina venezuelana e vírus mayaro (RAI, 1991; Consoli \& Lourenço-De-Oliveira, 1994). A transmissão transovariana dos vírus La Crosse e Santo Ângelo foi observada em Ae. albopictus (TESH, 1980; SHROYER, 1986). Nos EUA foi encontrada naturalmente infectada com vírus da encefalite tipo leste e no México pelos vírus da dengue 2 e 3 (Mitchell et al., 1992; IBÁÑEZBERNAL et al., 1997). A ocorrência deste culicídeo no Brasil (Rio de Janeiro) foi relatada por ForATTINI (1986) e vem se alastrando por vários estados brasileiros. O primeiro registro oficial de Ae. albopictus no Estado do Paraná foi feito por SANT'ANA (1996) em Curitiba, embora a presença no Estado fosse conhecida desde 1988, quando da identificação de algumas larvas encontradas em lotes de pneus no município de Arapongas, Paraná, através dos trabalhos de rotina da campanha anti-aegypti realizada pelos agentes da Superintendência de Campanhas de Saúde Pública, ex-SUCAM. Hoje ocupa toda a região norte e extremo oeste do Estado, estendendo-se para as áreas temperadas e do litoral paranaense.
Bambus e taquaras, monocotiledôneas da família das gramíneas apresentam colmo que favorece o acúmulo de água em seus entrenós, seja pela ação metabólica ou pela chuva armazenada em colmos abertos. Formam-se, nestes microhábitats, condições ideais para desenvolvimento de formas imaturas de culicídeos.

Considerando a capacidade do Aedes albopictus de colonizar criadouros naturais e artificiais, tanto da área urbana como áreas silvestres, tem-se como objetivo investigar entrenós de bambu e bromélias visando o diagnóstico da espécie na área de borda da Mata Atlântica, Paranaguá, Paraná.

\section{MATERIAL E MÉTODOS}

A planície litorânea do Estado do Paraná é uma estreita faixa de terra com largura entre 10 e $20 \mathrm{~km}$, tornando-se um pouco mais larga nas proximidades da Baía de Paranaguá, situada entre o Oceano Atlântico e as escarpas montanhosas da Serra do Mar, estendendose desde a Vila Ararapira, ao norte $\left(25^{\circ} 12^{\prime} 44^{\prime \prime}\right.$ S; $48^{\circ}$ $\left.01^{\prime} 15^{\prime \prime W}\right)$, até a Barra do Saí-Guaçu, ao sul (2558'38"; $\left.48^{\circ} 35^{\prime} 26^{\prime \prime W}\right)$. A vegetação litorânea, com uma área aproximada de $729 \mathrm{~km}^{2}$, é representada pelos mangues, pela vegetação das praias e pelos agrupamentos densos de vegetais das restingas, que embora não ocorrendo mais com a mesma densidade e extensão como nos litorais ao norte de São Paulo, Rio de Janeiro e Espírito Santo, muitas vezes possui árvores de 6 a 8 metros de altura, onde são observadas diversas variedades de pteridófitas e bromeliáceas terrestres e epífitas, com predomínio de palmáceas. Faixas de mata pluvial-tropical se estendem 
das montanhas até as restingas. O clima é tropical superúmido, com média anual de $21,1^{\circ} \mathrm{C}$, no mês mais quente superior a $21^{\circ} \mathrm{C}$ e no mais frio superior a $18^{\circ} \mathrm{C}$, sem estação seca e isento de geadas. A precipitação anual é em torno de $1976 \mathrm{~mm}$, sendo fevereiro o mais chuvoso e julho registrando o período de maior estiagem (MAACK, 1981; Wons, 1985).

A pesquisa foi realizada no povoado de ImbucuíMirim, no município de Paranaguá, Paraná (fig. 1). As coletas restringiram-se ao bambuzal e área circunvizinha localizada à margem de estrada secundária aberta dentro da mata e próxima a uma área degradada usada para extração de areia. $\mathrm{O}$ bambuzal foi cultivado pelos nativos da região que retiravam colmos para diferentes destinos. Ao cortar os colmos, restavam no solo as partes basais dos mesmos, cujos entrenós superiores ficavam abertos e expostos à água da chuva (fig. 2).

As coletas de larvas ocorreram em duas etapas, a primeira realizada em 23 de agosto de 2001, restringindose ao bambuzal e alguns recipientes naturais representados por bromélias epífitas localizadas num raio de 5 metros e de outras duas bromélias naturalmente fixadas em bambu. Na segunda etapa, em 4 e 5 de setembro de 2001, inspecionou-se uma área estimada em $3600 \mathrm{~m}^{2}$ em torno do bambuzal, onde foram pesquisadas bromélias

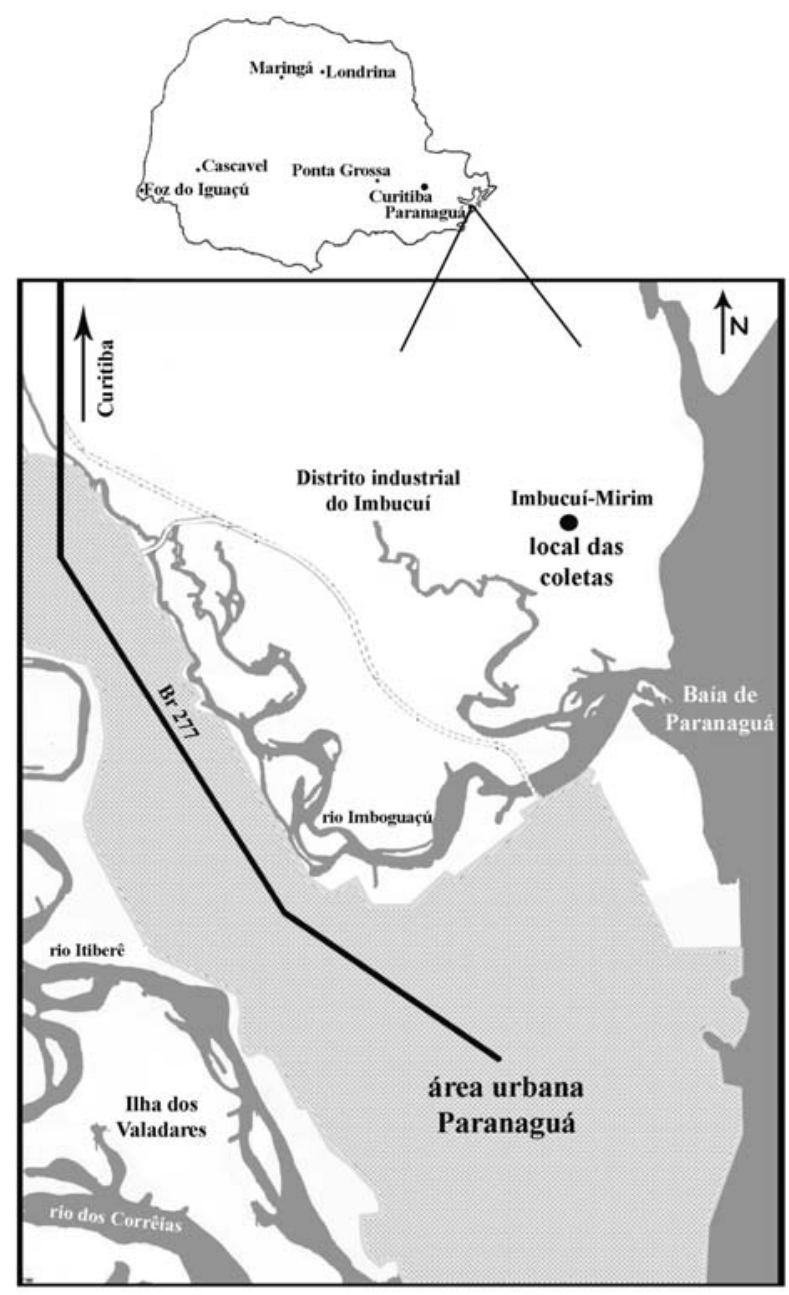

Fig.1. Localização da área de estudo no distrito de Imbucuí-Mirim, município de Paranaguá, Paraná.

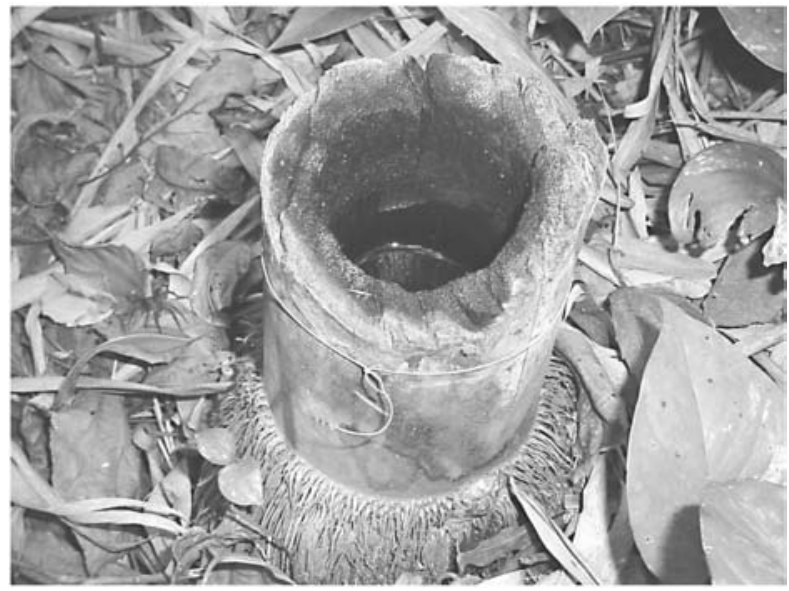

Fig. 2. Criadouro artificial de Culicidae originado pelo corte dos colmos de bambu.

epífitas e de solo e outras duas touças de Bambusa sp., sendo uma encontrada no interior da mata sem apresentar cortes em seus colmos e outra em área de extração de areia com vários colmos cortados. Para coletar as larvas nos entrenós, utilizou-se pipeta tipo conta-gotas. Com relação às bromélias, promoveu-se o emborcamento da planta até o escoamento da água contida nas imbricações de suas folhas numa bandeja, de onde foram retiradas as larvas. O volume da água foi medido através de proveta graduada. As larvas foram identificadas utilizando-se as chaves de identificação de ForATTINI (2002). Uma amostra dos imaturos foi introduzida em potes de vidro de boca larga com água do próprio criadouro, para criação em laboratório e obtenção de adultos para comprovação da identificação específica. Amostra do material identificado está depositada no Laboratório de Entomologia, Universidade Estadual de Londrina, Paraná.

\section{RESULTADOS E DISCUSSÃO}

Foram coletados 251 imaturos de mosquitos, sendo 210 obtidos em 18 bambus cortados: 100 Limatus durhami Theobald, 1901 (47,6\%), 95 Ae. albopictus (45,2\%) e 15 Culex (Microculex) sp. (7,2\%) (tab. I). Em 65 bromélias estudadas, entre aquelas fixadas no solo e até dois metros de altura, coletaram-se 41 espécimens, sendo 20 larvas de Culex (Microculex) spp. (48,8\%), 20 Wyeomyia spp. $(48,8 \%)$ e um Toxorhynchites sp. $(2,4 \%)$.

Inspecionaram-se 31 entrenós de bambus cortados contendo água, dos quais 18 apresentaram larvas de mosquitos (tab. I). A maior frequiência de larvas foi observada nos entrenós com volume de água entre 151 e $200 \mathrm{ml}$, enquanto que a maior freqüência de entrenós negativos ocorreu entre aqueles que armazenavam menor volume de água, 7,5 a $50 \mathrm{ml}$ (fig. 3). O volume de água dos bambus positivos variou de 30 a $260 \mathrm{ml}$ e Ae. albopictus ocupou $55,5 \%$ deles, sendo que em três observações se encontrava associada a Li. durhami (tab. II). As larvas dessas espécies são vistas explorando os mesmos tipos de ecótopos, sejam eles naturais, como entrenós de bambu, buracos em árvores, axilas de folhas e cascas de frutos, ou artificiais, representados pelos recipientes abandonados na floresta e no ambiente antrópico 
(Consoli \& Lourenço-De-Oliveira, 1994; Albuquerque et al., 2000; Silva, 2002). Para Forattini et al. (1998), bromélias encontradas no ambiente antrópico, junto às habitações, são consideradas como criadouros artificiais, uma vez que são usadas para fins decorativos, e nestes locais registraram Ae. albopictus.

Aedes albopictus foi a segunda espécie mais abundante nos entrenós de bambus, depois de $L i$. durhami. Considerando que o bambu seja um típico criadouro natural da primeira espécie, pode-se supor que

Tabela I. Larvas de Culicidae coletadas em entrenós de bambus e em bromeliáceas em Imbucuí-Mirim, Paranaguá, Paraná, em 23 de agosto e 4, 5 de setembro de 2001.

\begin{tabular}{|c|c|c|c|}
\hline Data & Criadouro & Espécie & $\mathrm{N}^{\circ}$ \\
\hline \multirow[t]{2}{*}{ 23.VIII.2001 } & Bambu & Li. durhami & 87 \\
\hline & & Ae. albopictus & 6 \\
\hline \multirow[t]{6}{*}{ 4, 5.IX.2001 } & Bambu & Ae. albopictus & 2 \\
\hline & & Cx. (Microculex) sp. & 1 \\
\hline & & Li. durhami & 1 \\
\hline & Bromélia & CX. (Microculex) spp. & 20 \\
\hline & & Wyeomyia spp. & 2 \\
\hline & & Toxorhynchites sp. & \\
\hline \multirow[t]{5}{*}{ Subtotais } & & Li. durhami & 100 \\
\hline & & Ae. albopictus & 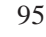 \\
\hline & & Cx. (Microculex) spp. & 3 \\
\hline & & Wyeomyia spp. & 2 \\
\hline & & Toxorhynchites sp. & \\
\hline Total geral & & & 251 \\
\hline
\end{tabular}

tenha vantagens sobre espécies nativas que também colonizam o bambu nesta região. As formas imaturas de Ae. albopictus possuem como microhábitats naturais ocos de árvores e entrenós de bambus, além de uma grande diversidade de recipientes artificiais (HAWLEY, 1988; LOPES, 2002). No Brasil, como espécie fitotelmata, foi encontrada em ocos de árvores e entrenós de bambus (Gomes \& Marques, 1988; Gomes et al., 1992) e em bromélias (FerReIRA Neto et al., 1987; NATAl et al., 1997; ForATTINi et al., 1998). Em bambus, restringem-se a recipientes cortados e introduzidos no ambiente, utilizados como armadilhas, no intuito de verificar a tendência desta espécie de usá-lo como criadouro (Gomes et al., 1992). Esta espécie foi registrada por AlBUQUERQUE et al. (2000) colonizando bambus e pneus em um remanescente da Mata Atlântica da área urbana de Recife, Pernambuco.

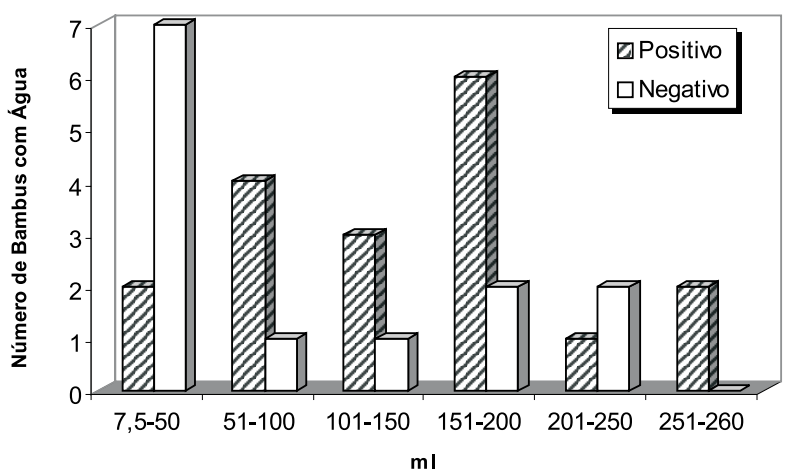

Fig. 3. Frequiência de entrenós de bambus encontrados com diferentes volumes de água, positivos e negativos para larvas de culicídeos, em Imbucuí-Mirim, Paranaguá, PR, em 23 de agosto e 4, 5 de setembro de 2001.
Tabela II. Variação do volume de água do interior dos entrenós de bambu e percentuais de positividade para larvas de mosquito, em Imbucuí-Mirim, Paranaguá, nas coletas realizadas em 23 de agosto e 4, 5 de setembro de 2001 .

\begin{tabular}{lccr}
\hline Espécies & $\begin{array}{c}\text { Volume de água } \\
(\mathrm{ml})\end{array}$ & $\begin{array}{c}\mathrm{N}^{0} \text { de entrenós } \\
\text { Positivos }\end{array}$ & $(\%)$ \\
\hline Ae. albopictus & $30-254$ & 7 & 38,9 \\
Ae. albopictus/Li. durhami & $200-260$ & 3 & 16,7 \\
Cx. (Microculex) spp. & $75-175$ & 3 & 16,7 \\
Li. durhami & $40-180$ & 5 & 27,7 \\
\hline Total & $30-260$ & 18 & 100,0 \\
\hline
\end{tabular}

Limatus durhami é conhecida pela alta valência ecológica, capaz de colonizar diferentes tipos de ecótopos e criadouros, principalmente aqueles introduzidos no ambiente, onde geralmente é a primeira a se instalar, mesmo naqueles que apresentam alto teor de matéria orgânica (GALINDO et al., 1951; LOPES; ARIAS \& Charlwood, 1985; Lopes; Charlwood \& Arias, 1985; Lopes et al., 1987). Embora suas larvas não sejam obrigatoriamente predadoras, como alguns sabetíneos, comportam-se desta maneira principalmente na ausência de alimentos no criadouro (LOPES ARIAS \& CHARLwOOD, 1985). Em um entrenó, onde as duas espécies coexistiam, registrou-se a maior densidade de Ae. albopictus. BARRERA (1996) diz que a rapidez do desenvolvimento e o grau de sobrevivência das larvas de Ae. albopictus estão diretamente ligados à disponibilidade de alimentação natural. Conforme a capacidade competitiva, alguma população de vetor emergente poderá ter acesso a nicho ecológico anteriormente ocupado por outra, que no caso é justificado pelo seu apreciável poder invasivo (ForatTini, 1998).

As espécies do subgênero Microculex normalmente são encontradas colonizando criadouros representados por recipientes naturais de caráter permanente, principalmente bromélias, mas também têm sido registradas em buracos de árvores e entrenós de bambu (ForATtini, 1965). ForatTini (1962) classificou bambus e taquaras cortadas como recipientes artificiais de caráter transitório. Aceitando esta classificação, o subgênero Microculex, aqui coletado, estava colonizando recipiente artificial de caráter transitório. De acordo com a revisão da classificação dos tipos de criadouros feita por FORATTINI (2002), espécies nativas de bambus ou taquaras que apresentam seus entrenós furados pela ação de animais ou outras circunstâncias, são classificados como naturais, todavia qualquer criadouro originado pela ação antrópica é considerado como artificial, mesmo estando em ambiente silvestre ou esculpido na vegetação nativa. Assim como os bambuzais, a taquara nativa está sob a ação de cortes sucessivos por parte do homem, o que acarreta na permanência da base do colmo, muitas vezes aberto em sua parte superior, o que permite a retenção de água da chuva. Esta nova condição pode contribuir para adaptação de Ae. albopictus à área de Mata Atlântica.

Na primeira etapa da pesquisa, os resultados obtidos em bromélias epífitas, do solo e para aquela fixa nos colmos de bambu, foram negativos para larvas de Culicidae. $\mathrm{Na}$ segunda etapa, nas 65 bromélias estudadas, coletaramse 41 larvas de Culicidae (tab. I). Também não foram constatadas larvas no bambuzal do interior da mata, que 
não apresentaram sinais de cortes, ao contrário daqueles localizados próximos às margens da estrada secundária que apresentavam diversos colmos cortados. Diante disto, Ae. albopictus parece acompanhar o homem na sua penetração na mata, contrariando a informação de Albuquerque et al. (2000), que consideram a possibilidade deste mosquito dispersar de área de mata para a área urbana de Recife.

Existe ainda a possibilidade da utilização de Bromeliaceae como criadouros para Ae. albopictus, fato já observado na região em 1997 com o registro de duas larvas de Ae. albopictus em bromélia epífita junto à comunidade de Amparo, município de Paranaguá (Álvaro C. Gonsalves, FUNASA/PR, comunic. pess.). A colonização das bromélias poderia levar à expansão da espécie para áreas mais protegidas, como o complexo montanhoso, constituindo-se em mais uma preocupação do ponto de vista epidemiológico (NATAL et al., 1997), pois neste ecótopo circulam arbovírus patogênicos ao homem (IVERSSON, 1988).

FORATTINI et al. (1990) descreveram áreas próximas e adjacentes ao meio natural primitivo, representado pela Mata Atlântica da planície, como o ambiente formado pelo homem que insere a criação de novos nichos ecológicos passíveis de serem ocupados por populações de culicídeos silvestres. IvERSSON et al. (1994) afirmaram que áreas protegidas da Serra do Mar, dada a sua complexidade ecológica de vertebrados e artrópodes hematófagos, podem favorecer a manutenção e transmissão de arbovírus, sendo as bordas desses ecossistemas áreas de risco a infecções acidentais.

Agradecimentos. Ao Dr. Nicolas Degallier, ORSTOM, Brasília, DF, pelas sugestões e aos agentes de saúde Almir Filadelfo Martins, 1ํㅡ Regional de Saúde e José Eduardo de Oliveira, 17 Regional de Saúde, Paraná, pelo apoio durante as coletas de campo.

\section{REFERÊNCIAS BIBLIOGRÁFICAS}

Albuquerque, C. M. R. De; Melo-Santos, M. A. V. et al. 2000. Primeiro registro de Aedes albopictus em área da Mata Atlântica, Recife, PE, Brasil. Revista de Saúde Pública, São Paulo, 34(3):314, 315.

BARRERA, R. 1996. Competition and resistance to starvation in larvae of container-inhabiting Aedes mosquitoes. Ecological Entomology, Oxford, 21:117-127.

Consoli, R. A. G. B. \& Lourenço-de-Oliveira, R. 1994. Principais mosquitos de importância sanitária no Brasil. Rio de Janeiro, Fiocruz. 225p.

Ferreira Neto, J. A.; Lima, M. M. \& Aragão, M. B. 1987. Primeiras observações sobre o Aedes albopictus no Estado do Espírito Santo, Brasil. Cadernos de Saúde Pública, Rio de Janeiro, 3(1):56-61.

Forattini, O. P. 1962. Entomologia Médica. São Paulo, USP. v.1, $662 \mathrm{p}$.

— 1965. Entomologia Médica. São Paulo, USP. v.2, 506p.

—. 1986. Identificação de Aedes (Stegomyia) albopictus (Skuse) no Brasil. Revista de Saúde Pública, São Paulo, 20(3):244, 245.

1998. Mosquitos Culicidae como vetores emergentes de infecções. Revista de Saúde Pública, São Paulo, 32(6):497-502. 2002. Culicideologia médica. São Paulo, USP. v.2, 860p.

Forattini, O. P; Gomes, A. DE C. et al. 1990. Freqüência ao ambiente humano e dispersão de mosquitos Culicidae em área adjacente à Mata Atlântica primitiva da planície. Revista de Saúde Pública, São Paulo, 24(2):101-107.
Forattini, O. P.; Marques, G. R. A. et al. 1998. Significado epidemiológico dos criadouros de Aedes albopictus em bromélias. Revista de Saúde Pública, São Paulo, 32(2):186-188.

Galindo, P.; Carpenter, J. \& Tropido, H. 1951. Ecological observations on forest mosquitoes of on endemic yellow fever area in Panama. American Journal of Tropical Medicine and Hygiene, Baltimore, 31:98-137.

Gomes, A. De C.; Forattini, O. P. et al. 1992. Microhabitats de Aedes albopictus (Skuse) na região do vale do Paraíba, Estado de São Paulo. Revista de Saúde Pública, São Paulo, 26(2):108-118.

Gomes, A. DE C. \& Marques, G. R. A. M. 1988. Encontro de criadouro natural de Aedes (Stegomyia) albopictus (Skuse), no Estado de São Paulo, Brasil. Revista de Saúde Pública, São Paulo, 22:(3)245.

Hawley, W. A. 1988. The biology of Aedes albopictus. Journal of the American Mosquito Control Association, Lake Charles, 4:2-39.

IBÁÑEZ-BERnAL, S.; BRICEÑo, B. et al. 1997. First record in America of Aedes albopictus naturally infected with dengue virus during the 1995 outbreak at Reynosa, Mexico. Medical and Veterinary Entomology, London, 11:305-309.

Iversson, L. B. 1988. Rocio encephalitis. In: Monath, T. P. Arboviruses: epidemiology and ecology. Boca Raton, CRE. v. 4, p.77-92.

Iversson, L. B.; NAtal, D. et al. 1994. Estudo da fauna de culicídeos com vistas ao isolamento de arbovírus em área de reserva natural do sistema Serra do Mar. Revista de Patologia Tropical, Goiânia, 23 (Suppl. 2):221.

Lopes, J. 2002. Mosquitos (Diptera: Culicidae) da região do baixo Tibagi e suas adaptações a ambientes antropogênicos: causas e consequiências. In: Medri, M. E. et al. ed. A bacia do rio Tibagi. Londrina, M. E. Medri. cap. 19, p.327-351.

Lopes, J.; Arias, J. R. \& Charlwood, J. D. 1985. Estudo ecológico de Culicidae (Diptera) silvestres criando em pequenos recipientes de água em mata e em capoeira no município de Manaus - AM. Ciência e Cultura, São Paulo, 37(8):1299-1311.

Lopes, J.; Borsato, A. M. \& Pires, M. A. 1987. Entomofauna da Mata Godoy. I. Culicidae (Diptera) procriando-se em criadouros artificiais introduzidos na mata. Semina, Londrina, 8(2): 67-69.

Lopes, J.; Charlwood, J. D. \& Arias, J. R. 1985. Sobreposição de espécies de Culicidae (Diptera), criando em pequenos recipientes artificiais de água em floresta e capoeira no município de ManausAM. Revista Brasileira de Biologia, Rio de Janeiro, 45(4):609-613.

MAACK, R. 1981. Geografia física do Estado do Paraná. 2. ed. Rio de Janeiro, J. Olympio. 450p.

Mitchell, C.; NiebYlski, M. et al. 1992. Isolation of eastern equine encephalitis from Ae. albopictus in Florida. Science, Washington, 257:526, 527.

Natal, D.; Urbinatti, P. R. et al. 1997. Encontro de Aedes (Stegomyia) albopictus (Skuse) em Bromeliaceae na periferia de São Paulo, SP, Brasil. Revista de Saúde Pública, São Paulo, 31:517, 518.

RaI, K. S. 1991. Aedes albopictus in the Americas. Annual Review of Entomology, Stanford, 36:459-484.

Sant'AnA, A. L. 1996. Primeiro encontro de Aedes (Stegomyia) albopictus (Skuse) no Estado do Paraná, Brasil. Revista de Saúde Pública, São Paulo, 30(4):392, 393.

Shroyer, D. A. 1986. Transovarial maintenance of San Angelo virus in sequential generations of Aedes albopictus. American Journal of Tropical Medicine and Hygiene, Baltimore, 35:408-417.

Silva, A. M. 2002. Imaturos de mosquitos (Diptera, Culicidae) de áreas urbana e rural no norte do Estado do Paraná, Brasil. Iheringia, Sér. Zool., Porto Alegre, 92(4):31-36.

Tesh, R. B. 1980. Experimental studies on the transovarial transmission of Kunjin and San Angelo viruses in mosquitoes. American Journal of Tropical Medicine and Hygiene, Baltimore, 29:657-666.

Wons, I. 1985. Geografia do Paraná. 5. ed. Curitiba, Ensino Renovado. 172p.

Recebido em fevereiro de 2003. Aceito em novembro de 2003. ISSN 0073-4721 